\title{
Cross-cultural Communication Strategies Research of Socializing Apps During Internet Era Using TikTok as Example
}

\author{
Yuheng Wang ${ }^{1, \mathrm{a}, \dagger}$ Wuhong Chen ${ }^{2, \mathrm{~b}, \dot{\dagger}, *}$
}

\author{
${ }^{1}$ Communication University of China, Beijing, China \\ ${ }^{2}$ Jinan University, Guangzhou, Province Guangdong, China \\ *Corresponding author. Email: ${ }^{b}$ phaniephanie@stu2018.jnu.edu.cn \\ These authors contributed equally.
}

\begin{abstract}
At present, many software products are trying to achieve cross-cultural communication overseas, and TikTok has been considered a successful case. Against this background, this paper uses the encoding/decoding theory to study the experience of TikTok's remarkable success in cross-cultural communication. This paper mainly uses the method of case study and the method of comparative study to explore the different communication strategies of the app in China and the US and their commonalities during the communication process. Based on the encoding/decoding theory, this paper separately analyzes the app's communication advantages in the different stages of encoding, decoding, and circulation. The research directions and research methods selected in this paper are innovative and practical, which enriches the development of the encoding/decoding theory and expands its explanatory power and scope of application. At the same time, one of the most successful apps for cross-cultural communication, TikTok, is used as the research object to provide a reference for corporation operators of social apps and other transcultural communicators.
\end{abstract}

Keywords: TikTok, social apps, cross-cultural communication, encoding/decoding theory

\section{INTRODUCTION}

According to the 47th Statistical Report on Chinese Internet Development Condition released by the China Internet Network Information Center (CNNIC), the number of short-video users in China had reached 873 million by December 2020, $88.3 \%$ of all Internet users. At the same time, the figure increased by one hundred million from March of the same year [1]. By now, we can tell that short videos are still experiencing speed development. Among various short-video platforms, TikTok has shown great competitiveness: It has gained significant growth and remarkable results at home and abroad, especially since it was launched overseas in 2017. As Sensor Tower's intelligence data indicate, in December 2020, TikTok (both Douyin and its overseas version) attracted nearly $\$ 142$ million in the App Store and Google Play worldwide. The figure was 3.3 times that of last December, ranking the top again on the revenue chart of the world's mobile applications (nongame). More specifically, about $86 \%$ of its revenue came from the Chinese version (Douyin). The US market was the second largest (contributing 7\% of the total revenue), followed by the Turkish market (taking up 2\%) [2].

As a social app, TikTok has gained major success in the field of transcultural communication. In North America, TikTok has become the most successful Chinese mobile app through the acquisition and merger of its native competitors. In Japan, with the current situation of local enterprises' conservative holding together, TikTok has stood firm and successfully carried out localization transformation. In Southeast Asia, the precise marketing to young people has made many teenagers feel a sense of belonging and loyalty to TikTok. Nevertheless, previous studies tend to focus more on social apps' successful experience in a specific country or region but less on how they achieve cross-cultural communication in different cultural environments. Therefore, through analyzing the case of TikTok and its transcultural communication patterns, this research will help fill in the blanks.

Based on the previous discussion, this paper focuses on the following questions: What marketing and communication strategies have TikTok applied in China 
and the US? What difficulties has it encountered during its transcultural communication? What are TikTok's inherent advantages that have enabled it to achieve such great success in the field of cross-cultural communication? Considering that encoding and decoding theory is often used in the research of crosscultural communication, and has achieved remarkable results. This research will mainly apply the case study approach and comparative analysis approach based on coding and decoding theory to solve the above questions.

\section{LITERATURE REVIEW}

At present, many studies have focused on the topic of cross-cultural communication. Some of these studies analyze the elements of transcultural communication by adopting such communication theories as Edward Hall's theory of nonverbal communication, Hofstede's cultural dimensions theory, Gudykunst's anxiety/uncertainty management theory, etc. Meanwhile, from a practical perspective, some others analyze how certain TV series, movies, and apps attain cultural communication globally. Although many existing references are related to the academic theories and research achievements of crosscultural communication, this literature review will mainly focus on three topics: connections between the encoding/decoding theory and cross-cultural communication; the encoding/decoding theory's applications in cross-cultural communication case studies; TikTok's cross-cultural communication methods and effects.

An overview of the relationship between the encoding/decoding theory and cross-cultural communication. Stuart Hall, the British cultural theorist, put forward the encoding/decoding theory in his paper Encoding and Decoding in the Television Discourse. Many scholars believe that the encoding/decoding theory can be used in cross-cultural communication study. For instance, Wu Haiying believes that the essence of crosscultural communication is the process of "two-stage encoding" [3]. This is because the encoder and decoder are in different language systems. Therefore, both need a "translator" as an intermediary to encode and decode twice to complete the transcultural communication. However, Wang Shengnan, Xie Yuanyuan, and others believe that cross-cultural communication requires a highly equalized encoding process and decoding, especially when conducting large-scale dissemination among a large number of users [4].

An overview of the encoding/decoding theory's applications in cross-cultural communication case studies. At present, the encoding/decoding theory's application in case studies is mainly concentrated on TV dramas, movies, and other literary works. For instance, Liu Jiaxin, Jiang Huiman, and others use the drama Why Women Kill as a case to analyze the cross-cultural spread of American dramas in China, basing on the encoding/decoding theory [5]. They believe that American dramas' recoding in China is mainly carried out through plot symbols, line symbols, visual presentation symbols, etc. Moreover, the decoding process of the Chinese audience presents the dominanthegemonic mode and the negotiated mode. Li Ping, however, takes Kung Fu Panda 2 as an example to analyze the cross-cultural communication mode of movies. Li believes that encoders have dominant or preferential meaning during the encoding process. Such dominant meaning represents some sorts of ideologies and the encoders' core ideas and is a kind of communication framing [6]. At the same time, some scholars apply the theory of encoding and decoding to study other forms of transcultural communication. For example, Guo Jingyu studies the encoding and decoding process of brands in transcultural communication [7]. Guo believes that the text writing and translation should fully consider the recipient country's language system and aesthetic taste.

An overview of TikTok's cross-cultural communication methods and effects. TikTok has become a leader in the global short video market. The product receives great acclaim both at home and abroad. Its number of downloads and audience make it a favorable research object for many scholars engaged in crosscultural communication study. In China, many researches have been carried out on TikTok's overseas communication strategies and its advantages in crosscultural communication. For example, Li Xin, Li Desheng, and others analyze TikTok's overseas communication strategies. According to them, the key to TikTok's overseas success lies in adopting a localization strategy based on each market's actual situation and different characteristics [8]. What's more, Wei Yuhang believes that TikTok's content diversity and information inclusiveness bring it widespread popularity, and its usercentered function makes it a huge success overseas [9].

In conclusion, the above literatures mainly focus on two aspects: the relationship between the encoding/decoding theory and transcultural communication; TikTok's achievements of transcultural communication. It can be seen that the encoding/decoding theory can be used as the theoretical basis for cross-cultural communication case studies, and TikTok is an excellent case of cross-cultural communication. However, few literatures have combined them to study the success of TikTok's cross-cultural communication with the encoding/decoding theory. Therefore, based on the encoding/decoding theory, this study will fill in this gap and analyze TikTok's crosscultural communication achievements using the case study method and comparative analysis method. 


\section{AN OVERVIEW OF TIKTOK AND AN ANALYSIS OF ITS CROSS-CULTURAL COMMUNICATION STRATEGIES: TAKING CHINA AND THE US AS EXAMPLES}

\subsection{An Overview of Social Apps' Development in the Internet Era}

With the rapid upgrading and proliferation of mobile Internet technology, new social media platforms are emerging one after another. The extensive popularity of mobile phones and the widespread use of Internet technologies provide the womb for newly born social software. Moreover, they are the carrier and channel of these apps during communication. At present, there are a lot of classifications for these social apps. Among them, the relatively representative five categories are Traditional social apps such as FaceBook, Twitter, and WeChat, where people can be connected with others. Blogging apps such as Tumblr and Weibo, which engage users by written content. Media sharing apps such as Instagram, YouTube, and Bilibili, featuring an image or even audio-visual contents. Discussion forums such as Quora and Zhihu, where users can share or discuss information and opinions. Finally, other apps including consumer review apps, shopping apps and even dating apps[10].

The rapid development of social apps has brought a lot of convenience to people's life. Nevertheless, the popularity of social apps is a double-sided sword. For one thing, it helps narrow down the distance between people. As instant messaging is becoming more convenient, people can contact their family and friends whenever and wherever they are. Even some people can reunite with their previous schoolmates through social apps, which was impossible decades before. However, indulging in social apps also brings problems. For example, netizens are getting used to texting with phones, and their interpersonal skills are weakened. There are also many other drawbacks of social platforms like cyberbullying and cyber fraud. In a word, social apps have both positive and negative impacts on people's daily life.

\subsection{An Overview of TikTok's Development History}

\subsubsection{Early-stage of Domestic Development}

As a famous social app in China, TikTok is owned by the Chinese information technology company ByteDance. The app went online in May 2017[11]. As ByteDance's new strategic product, TikTok has received great supports, both technologically and financially, from its parent company and experienced rapid growth. For example, TikTok uses the algorithmic content distribution mechanism of TouTiao [12], which is also ByteDance's core product (literally "Headlines").

2017 witnessed the year for TikTok. It soon became one of the two dominant short-video apps in China's domestic market along with Kuaishou. Even by now, TikTok still ranks first among its peer apps. According to App Annie's Top Apps on iOS, China, Overall, April 29, 2021, TikTok ranked eighth among free apps, while its counterpart Kuaishou ranked twenty-third[13].

\subsubsection{The Later Stage of Overseas Expansion}

Under the lead of ByteDance's founder Zhang Yiming, TikTok decided to expand its market overseas to gain a larger user scale. In November 2017, it purchased Musical.ly for US\$1 billion with the support of its parent company [14]and thus gained a foothold in the North American market. During its expansion, cultural differences and political risks are significant problems. In 2020, Donald Trump threatened to ban TikTok from US app stores for the sake of national security [15]. Although Joe Biden paused the ban, risks alike are still worth close attention.

However, TikTok is still an important force in the field of cross-cultural communication in China. According to the statistics provided by Business of Apps, TikTok topped App Annie's list of 2020's most downloaded apps [16], surpassing Facebook, Zoom Cloud Meetings, and Netflix during the year of pandemic.

\subsection{Comparison of TikTok's Cross-cultural Communication Strategies between China and the US}

TikTok is a successful social platform to set up overseas markets. The adaptability of its transcultural communication strategies basically lies in its content, product promotion, and algorithm.

\subsubsection{Content Production: UGC and Meme Theory}

TikTok features UGC (User-generated Content) in both China and overseas versions, and it takes advantage of Meme theory to help its growth and promotion [17]. In both China and America, TikTok users are encouraged to generate their short videos by imitating existing content. Replicators (original video contents) are usually catchy, easy to copy, and of high quality. Through the process of duplication and diffusion, certain video contents can go viral within a short period. The strategy lowers the threshold of content dissemination, and at the same time, improves user stickiness.

However, popular contents are not totally the same in China and the US. People of different cultural 
backgrounds tend to have different preferences. Therefore, simply applying the content operation model in China to the US version will not work out. In this regard, it adopts a localization strategy in the US for better promotion. For instance, TikTok implements a talent internationalization strategy by recruiting comprehensive talents with transcultural backgrounds and profound acquisition of local culture [18]. In this way, TikTok has created and promoted more culturerelated content in the US and open up new markets successfully.

Moreover, in China, TikTok provides KOL (influencers) with subsidies and other favorable policies to encourage them to produce quality content. Therefore, it guarantees the quantity as well as the quality of replicators.

\subsubsection{Content Promotion: The Combination of Online and Offline Channels}

TikTok combines online and offline promoting strategies to attract users in both China and the US. On the one hand, it makes good use of popular social media platforms. Existing platforms have already accumulated a large number of users. By cooperating with these platforms, TikTok is able to expose itself to more potential users. On the other hand, it has also conducted many offline campaigns to increase its influence. Nevertheless, although its promoting strategies in the two countries are theoretically the same, there are still some differences in practice.

Take online promotion as an example. In China, it cooperates with multi-media platforms. For instance, it exchanged and shared resources with Netease Cloud Music's music software to enhance its influence. What's more, TikTok also sponsored the variety show China's Got Hip-hop and integrated resources by inviting show contestants to join its platform [19]. However, in the US, TikTok focuses on such social media platforms as Instagram, Facebook, and YouTube [20]. These popular platforms have similar functions and user compositions with TikTok and can help it attract new participants.

\subsubsection{Content Distribution and Communication Effects: Meeting Users' Needs through Algorithm and Recommending Mechanism}

TikTok attributes much of its success to its recommending mechanism and the supporting algorithm behind it. In both China's and America's versions, the AI algorithm is used for content recommendation. Its major logic is to match users with potential content according to several factors. For example, users' thumbs-up, reposts, and comments will be recorded. These data can tell each user's preference. Accordingly, the platform will select and recommend different contents meeting their preference.
Based on Lasswell's " $5 \mathrm{~W}$ " communication mode, an act of communication includes the effects it generates. Also, communication activities are the sum of dissemination, acceptance, and feedback. Under TikTok's algorithm and recommending mechanism, users' interpretation and feedback on video content are analyzed and become the basis for distributing new content later. In this way, the different needs of each user are attended to and satisfied.

According to the comparisons made above, the pattern of TikTok's major communication strategies in the two countries are generally the same, with some differences in detail catering to their distinguished cultural backgrounds

\section{THE REASONS FOR TIKTOK'S SUCCESS IN CROSS-CULTURAL COMMUNICATION: AN ANALYSIS BASED ON THE ENCODING/DECODING THEORY}

\subsection{An Overview of the Encoding/Decoding Theory}

The Encoding-decoding theory was put forward by Stuart Hall in his paper Encoding and Decoding in the Television Discourse. As one of the outstanding researchers of British culture, Hall devoted his whole life to the study of mass media and mass culture. Meanwhile, he was also one of the founders of the Birmingham School of Cultural Studies.

As a well-known Marxist sociologist, Stuart Hall referred to Marxist Political Economy (MPE) theory and developed the Encoding-decoding theory. MPE has four basic sections_-production, circulation, distribution/consumption, reproduction. Based on this Marxist theory, the production and dissemination process of meaning in the television discourse was divided into three stages. The first stage is the "encoding" stage, where encoders' world perspectives predominate. In the second phase of "message", meaning is injected into the television discourse, and the works are completed. The second stage presents a discourse system of polysemy and openness, and its dominant factor is languages and rules of the speech that give meaning to television works. Finally, the "decoding" phase includes the audience's use and reproduction of information. The codes receivers (decoders) use to decode messages may differ from that of the senders (encoders). Therefore, the "meaning" decoded may not be so identical to what the senders have anticipated.

More specifically, resulting from personal knowledge and technical structure, TV audiences will develop different systems of symbols from different production relations. In the "decoding" stage, such systems are used to decode TV symbols. If the audiences have the same 
symbol system as TV producers, content meaning can be conveyed without bias; however, if they possess different symbol systems, there can be deviations in the decoding process. Nevertheless, every society and culture is isolated to various degrees. In general, content construction and the cohesion of codes are influenced by many factors, and misunderstandings tend to exist.

At the same time, Hall suggested three hypothetical positions of decoding, including the dominanthegemonic position, the negotiated position, and the oppositional position. For news purveyors, the ideal mode of communication is to have the audiences decode within the range of the dominant code. In this perspective, the way and manner of interpreting information are completely in line with the expectations of the encoding process. However, the audiences may be unwilling to adhere to the path of dominant codes and interpret the implication as anticipated by information senders. For this reason, we have the dominanthegemonic code and the oppositional code. By contrast, however, the negotiated code seems more open and inclusive. Within a stage of negotiated code, purveyors can successfully disseminate messages, whereas information receivers can assert their unique rights for personal interpretation.

\subsection{An Analysis on the Reasons for TikTok's Success in Cross-cultural Communication: From the Perspective of the Encoding/Decoding Theory}

TikTok is considered a market leader of the shortvideo era, and its successful operation mode may be preferable for other social apps' transcultural communication. Therefore, this paper seeks to interpret TikTok's transcultural content production and dissemination process, based on the Hall Theory of Encoding and Decoding.

\subsubsection{Stage of Encoding}

According to Hall's theory, the process of encoding consists of content construction and the cohesion of code. What's more, as diverse cultures and societies tend to close the communication, such a process receives interference from various sorts of factors. Nonetheless, TikTok's production mode of User-generated Content (UGC) has done well to avoid the impact of the cultural enclosure. In different countries, the platform provides different filming and editing tools for local content producers to promote content indigenization. Meanwhile, most short video contents are produced independently or cooperatively by local residents, adopting the audiovisual language catering to local culture. As the encoders and decoders share similar cultural and social backgrounds, they are also more likely to share a similar symbol system. Therefore, the realization of unbiased meaning is made possible through TikTok.

At the same time, short videos feature both audio and visual functions simultaneously and thus have better communication effects than merely written languages. In the Anthropology of Manners, Edward Hall put forward the notion of nonverbal communication, suggesting that tone, gesture, expression, and time can all implicate the cultural psychology of transcultural communication. With contents disseminated in the form of short videos, TikTok has lowered the threshold for literacy. Therefore, both encoding and decoding have become much easier. As a result, the process of dissemination is accelerated.

\subsubsection{Stage of Circulation}

During the finished "message" stage, Hall believed content construction and circulation were under the impact of many kinds of factors. To reduce the influence of various restrictive factors, TikTok spares no effort in content promoting and disseminating. Its promotion mode combines online and offline activities together to improve the depth and breadth of original content communication. Meanwhile, TikTok has set up and maintained a partnership with many major video websites. For example, users can share their videos on the platform and other non-professional short video websites like YouTube (in America) and Bilibili (in China). If users are interested, they can also share the videos with their friends and family through other social apps like FaceBook (in America) and WeChat (in China). Such measures can minimize the possible impact posed by multi-media during dissemination.

At the same time, TikTok's combination of online and offline marketing reduces the resistance of its content communication. For example, its offline "Better Life Festival" activity held in 2019 gained over 6 billion play volume, with more than 20,000 people punching in. What's more, TikTok announced its cooperation with Oracle in the US to support the precise delivery of its content. These efforts reduce the interference of TikTok's content construction and circulation and enhance its communication capability.

\subsubsection{Stage of Decoding}

Hall divided the decoding mode into the dominanthegemonic mode, the negotiated mode, and the oppositional mode. The dominant decoding mode means that the audience will fully accept the opinions of the encoder. In contrast, the oppositional decoding mode means that the recipient will deliberately misinterpret the intention of the encoder. Both of these two decoding modes will reduce the communication efficiency of the encoded information and limit the audience's space for free interpretation. 
During TikTok's content dissemination process, the audiences decode information in a negotiated position. In this mode, after watching a video on the platform, the audience can choose whether to like, favor, or share it according to their own experience. They can also interact with the author and even others watching this video through the comment area. The negotiated mode prevents the decoding process from becoming encoders' one-way transmission. What's more, the audience is encouraged to understand more profoundly and actively what the author wants to express, and they also have the opportunity to put forward different opinions. In this mode, the user experience is satisfied to a large extent.

Meanwhile, in order to get more platform recommendations and thus likes from the audience, authors tend to listen more to the audience's voice, refer to fans' opinions, and consciously produce video content to meet their preferences. This not only provides highquality content for the platform but also attracts and maintains users. Also, authors can gain benefits and thus build up a win-win situation between them and the platform. It can be seen that compared to the other two modes, the negotiated mode is more in line with TikTok and other entertainment-oriented social apps alike. This mode increases the user's sense of participation, provides a better user experience, and improves user stickiness.

\subsection{Suggestions for Social Apps on Cross- cultural Communication Development}

First, social apps should focus on localizing the content encoded during transcultural communication and attending cultural, ethnic, and religious differences. For example, in July 2018, Indonesia banned TikTok for "inappropriate content and blasphemy"[21], but the ban was overturned a week later when it agreed to censor its "negative content". Therefore, it is suggested that they align their business modes with local policies and keep their content production aligned with local culture, customs, and habits. What's more, TikTok's UGC mode is also referable.

Meanwhile, such social apps should also pay attention to the variety of content forms, especially visual content presentation. They can also improve content's communication capabilities through audio and visual functions. In the case of TikTok, it provides its users with many fascinating filters, shooting templates, and catchy music clips. Its ability to produce audio-visual content caters to the audience's needs. At the same time, these functions encourage influencers to create more duplicable content for other users to imitate and thus improve the user stickiness.

Finally, social apps should be considerate for the audiences. This includes their efforts to reduce the decoding process's difficulties and enhance the effects of the negotiated mode. That is to say, they can set up a commentary area and emphasize the importance of thumbs-up and favorites to force the platform's contents to upgrade. What's more, TikTok has made some optimizations and innovations for user interaction. For instance, users can double-click any blank space on the screen instead of looking for the thumb-up button while watching videos [22]. Such endeavor indicates that TikTok attaches great importance to user experience.

\section{CONCLUSION}

On the whole, there are two deficiencies in this paper. First, due to the difficulty in collecting TikTok's data and background, this paper mainly adopts secondary data, which restricts the analysis depth of the paper to some extent. Also, because the encoding/decoding theory is rooted in western culture and was originally used for the communication analysis of TV media, its explanatory power may be diminished in conducting the crosscultural communication research of social apps in the Chinese context.

Despite the above, however, the research directions and research methods selected in this paper are still innovative and practical. At present, there are few research results on cross-cultural communication of social apps, and few have included them in the current research system from a theoretical perspective. At the same time, although the encoding/decoding theory is considered by many to be an important tool for crosscultural communication, there has been little practice. Using the encoding/decoding theory to explain the crosscultural communication of social apps, this article makes up for these two aspects of research deficiencies, enriches the connotation and extension of the encoding/decoding theory, and expands its theoretical explanatory power and scope of application. In addition, this paper adopts the method of the case study. To use TikTok, one of the most successful apps for transcultural communication, as the research object has strong practical significance.

Overall, based on the comparison of TikTok's communication strategies in China and the US, this paper analyzes TikTok's development history, current situation and analyzes its cross-cultural communication advantages with the encoding/decoding theory. This paper provides a reference for corporation operators and cultural disseminators. On the one hand, they can draw on the advanced experience of TikTok's cross-cultural communication; on the other hand, they can reconsider how to provide users with more convenient and innovative services from the perspective of the encoding/decoding theory.

\section{REFERENCES}

[1] 47th Statistical Report on Chinese Internet Development Condition, the China Internet Network Information Center (CNNIC) 
[2] https://www.yicai.com/news/100908717.html

[3] H. Wu, Application and development of the encoding/decoding theory in cross-cultural communication, in: Journalism Lover, vol. 2010(02):4-5. DOI:

10.16017/j.cnki.xwahz.2010.02.038

[4] S. Wang, Y. Xie, Analyzing the cross-cultural communication of TikTok based on the encoding/decoding theory, in: Public Communication of Science \& Technology, vol. 2020,12(19):127-129. DOI: 10.16607/j.cnki.16746708.2020 .19 .040

[5] J. Liu, H. Jiang, A brief analysis of the cross-cultural communication of American TV dramas in China based on the encoding/decoding theory: Using "Why Women Kill" as an example, in: Radio \& TV Journal, vol. 2021(01):47-49. DOI: 10.19395/j.cnki.1674-246x.2021.01.022

[6] P. Li, Cross-cultural communication of "Kung Fu Panda 2" from the perspective of the encoding/decoding theory, in: Journal of News Research, vol. 2019,10(09):219-220.

[7] J. Guo, An analysis of national image in brand crosscultural communication: Based on Stuart Hall's encoding/decoding theory, in: Public Communication of Science \& Technology, vol. 2020,12(22):84-86. DOI: 10.16607/j.cnki.16746708.2020.22.029

[8] X. Li, D. Li, A research on overseas communication strategies of TikTok short-video: Taking the US, Japan and Southeast Asia for example, in: Journal of Beijing Institute of Graphic Communication, vol. 2021,29(01):5-7. DOI: 10.19461/j.cnki.10048626.2021.01.002

[9] Y. Wei, The development and cross-cultural communication of social apps in the Internet era: Using TikTok as an example, in: Today's Massmedia, vol. 2020,28(04):50-51.

[10] https://www.digitalvidya.com/blog/types-of-social$\underline{\text { media }}$

[11] https://www.bytedance.com/zh/, date of visit

[12] H. Li, Research on the propagation dependence of TikTok, in: China Newspaper Industry, vol. 2018(02):45-46. DOI: 10.13854/j.cnki.cni. 2018.02. 022

[13]https://www.appannie.com/en/apps/ios/top/china/ov erall/iphone/

[14] https://www.bbc.com/news/technology-50267985

[15] https://www.nytimes.com/2020/09/18/business/trum p-tik-tok-wechat-ban.html
[16] https://www.businessofapps.com/data/tik-tokstatistics/

[17] J. Chang, H. Tian, The short-video culture from the perspective of meme theory: Based on the case study of TikTok, in: News and Writing, vol. 2018(12):32-39.

[18] B. Liao, Y. Zhang, Overseas market operation and inspiration of TikTok, the short-video media, in: Chinese Culture and Communication Studies, vol. 2020(02):178-189.

[19] J. Wu, Why short-video apps for music and social networking have become popular: Taking TikTok as an example, in: New Media Research, vol. 2017,3(18):88-89. DOI: 10.16604/j.cnki.issn20960360.2017 .18 .035

[20] B. Liao, Y. Zhang, Overseas market operation and inspiration of TikTok, the short-video media, in: Chinese Culture and Communication Studies, vol. 2020(02):178-189.

[21] https://www.reuters.com/article/us-indonesiabytedance-ban-idUSKBN1JU0K8

[22] H. Li, Research on the propagation dependence of TikTok, in: China Newspaper Industry, vol. 2018(02):45-46. DOI: 10.13854/j.cnki.cni.2018.02. 022 